\title{
Challenges of organic "cocrystals"
}

\author{
Weigang $\mathrm{Zhu}^{1,2}$, Huanli Dong ${ }^{1^{*}}$, Yonggang $\mathrm{Zhen}^{1}$ and Wenping $\mathrm{Hu}^{{ }^{*}}$
}

Organic cocrystal (also "co-crystal"), formed with two or more different components via non-covalent intermolecular interactions, possesses novel, unpredicted and unique properties, which are not the simple sum of those molecular components, e.g., with effect of $1+1>2$. In this regard, organic cocrystals provide a distinctive strategy for the synthesis of novel multifunctional materials, and an important platform for exploring new fundamental physicochemical phenomena in molecular systems, such as high conductivity $[1,2]$, ambipolar charge transportation [3], photovoltaic behavior [4-6], white light-emitting [7], room-temperature phosphorescence [8], nonlinear optics [9-12] and ferroelectricity [13] etc., with potential application even in liquid crystal engineering [14,15] and drug industry [16].

Probably, the first cocrystal could be tracked back to 1844 assigning to the discovery of "quinhydrone" by Wöhler [17], while the conception of "cocrystal" was firstly used by Schmidt and Snipes [18] to describe the crystal of pyrimidine and purine complex in 1967. However, the widely acceptance of this concept is still progressing, since the definition of a cocrystal has been debated in the crystallography field. Another terminology of "molecular complex" was also proposed by Patrick and Prosser [19] to define the mixture of benzene and hexafluorobenzene in 1960, and then became another word to describe crystals with multi-components. The word cocrystal re-attracted attention since 2003, and a generally accepted definition is a structurally homogeneous crystalline material that contains two or more neutral building blocks that are present in definite stoichiometric amounts [20,21]. We have gathered and analyzed all reported organic cocrystals, and divided them into four types according to the intermolecular interactions responsible for the co-crystallization: halogen-bonded [22], hydrogen-bonded [23], $\pi-\pi$ packing [24,25] and charge transfer (CT) [26]. Using this kind of division, a much clear image of organic cocrystals could be given. Molecules in cocrystal pack general in two modes: i) mixed-stacking, wherein different molecules alternative- ly pack in a DADA...DADA fashion with sufficient D-A $\pi$-electron overlap, ii) segregated-stacking, wherein molecules pack in a DDDD...AAAA fashion with D-D and A-A $\pi$-overlap.

As a novel research area, there are full of challenges for organic cocrystals. The first challenge is the basic mechanism of co-crystallization, because not any two types of materials can recognize each other and self-assemble into a cocrystal. Hence, a question is what kind of material is suitable for co-crystallization, and how different molecules co-assemble, nucleate and crystallize. This is also related to the method applied for co-crystallization, including solution [24], vapor-phase [6,27-29] and mechanochemical techniques $[16,30]$. In our experience, some fundamental conditions are also essential for solution co-crystallization, such as strong intermolecular interactions, planar molecular structure and similar solubility of donor (D) and acceptor (A). In order to reveal the nucleation and growth mechanism of organic cocrystals and further control the self-assembling process of co-crystallization, we used perylene-tetracyanoquinodimethane (TCNQ) CT cocrystal as an example [31], and found that the morphology and phase of cocrystals mainly depend on the concentration of perylene $\left(C_{\text {perylene }}\right)$ rather than TCNQ $\left(C_{\mathrm{TCNQ}}\right)$, and high $C_{\text {perylene }}$ is favourable for the formation of (perylene) ${ }_{3}$ TCNQ (P3T1) microblocks, while low $C_{\text {perylene }}$ faciliates the formation of (perylene) ${ }_{1}$ TCNQ (P1T1) nanowires (Fig. 1). More importantly, we demonstrate that the formed D-A complex is regarded as a new material species, because the solubility of the formed P3T1 in acetonitrile is obviously lowered than that of any single components. It actually allows us to define the cocrystal as a new molecular species. The structure-function relationship of these cocrystals is further investigated. P1T1 cocrystals exhibit n-type behavior with electron mobility of $0.05 \mathrm{~cm}^{2} \mathrm{~V}^{-1} \mathrm{~s}^{-1}$, while P3T1 cocrystals display ambipolar behavior with high hole mobility of 0.03 $\mathrm{cm}^{2} \mathrm{~V}^{-1} \mathrm{~s}^{-1}$ and low electron mobility of $2.1 \times 10^{-5} \mathrm{~cm}^{2} \mathrm{~V}^{-1} \mathrm{~s}^{-1}$. Moreover, white light responsivity of P1T1 is higher than

\footnotetext{
${ }^{1}$ Institute of Chemistry, Chinese Academy of Sciences, Beijing 100190, China

${ }^{2}$ University of Chinese Academy of Sciences, Beijing 100049, China

* Corresponding authors (emails: huwp@iccas.ac.cn (Hu W); dhl522@iccas.ac.cn (Dong H))
} 
a

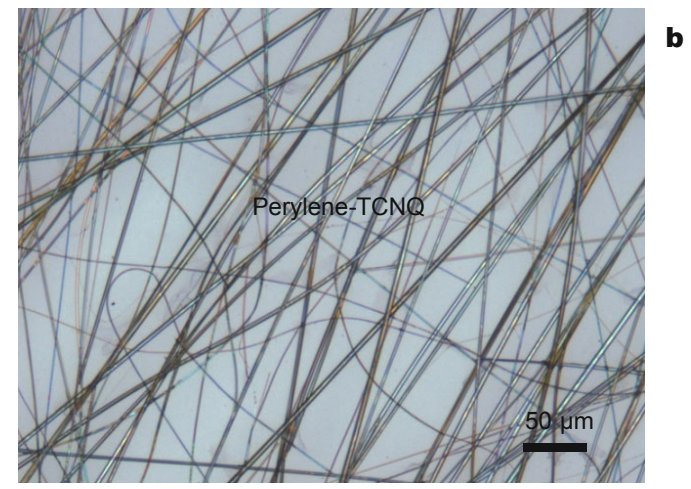

c

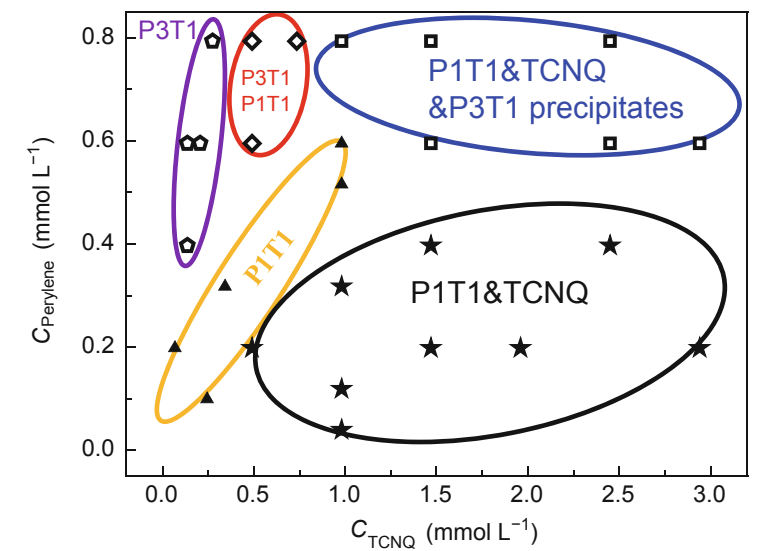

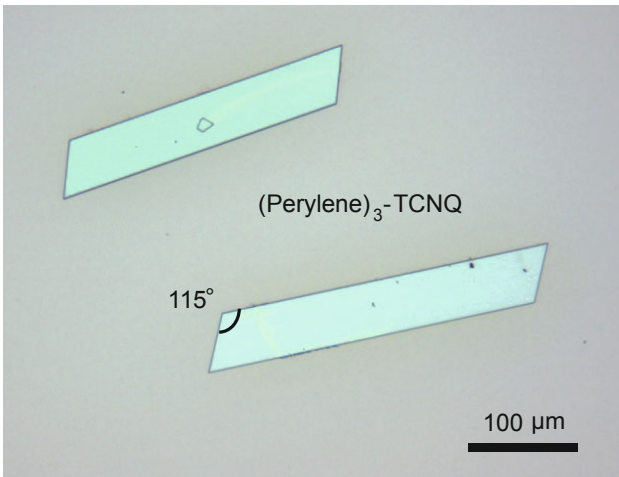

d

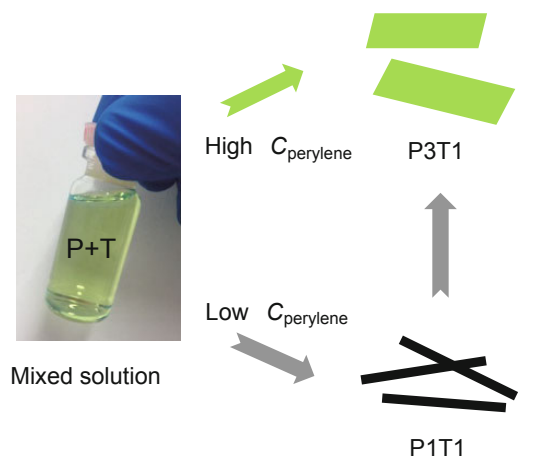

e
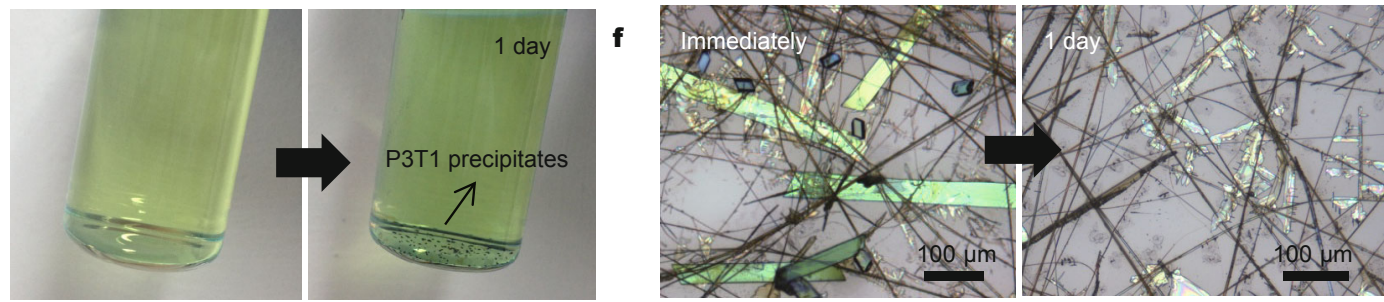

g

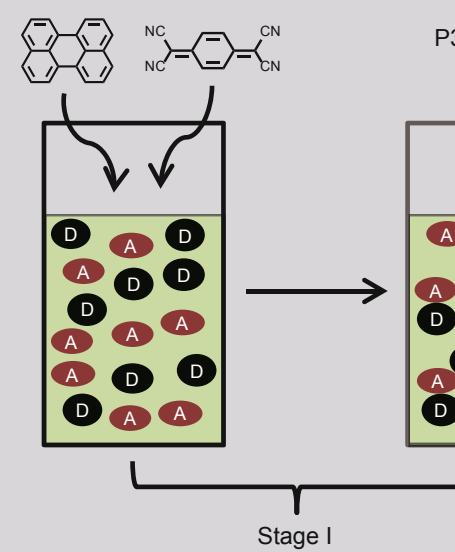

D-A complex formation

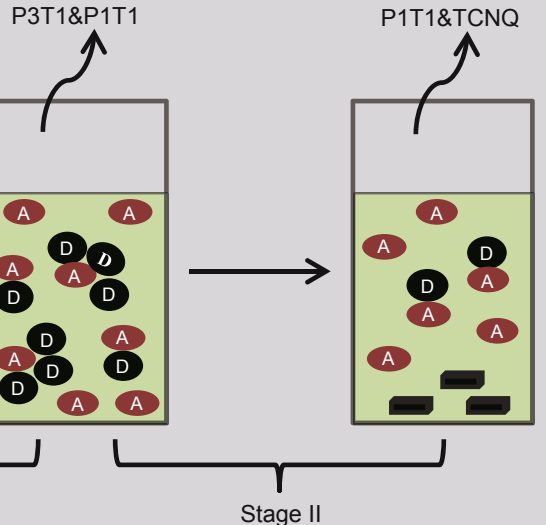

P3T1 crystal precipitation

Figure 1 Optical images of (a) P1T1 nanowires and (b) P3T1 microblocks. (c) The dependence of crystal morphology and material concentration in acetonitrile solution. (d) Schematic illustration of the formation mechanism of cocrystals. (e) The optical images of mixed solution $\left(C_{\text {perylene }}=0.80\right.$ $\mathrm{mmol} \mathrm{L}-1, C_{\mathrm{TCNO}}=2.45 \mathrm{mmol} \mathrm{L}^{-1}$ ) prepared immediately and after aging for one day. (f) The optical images of the obtained crystals from the corresponding solution after aging for different time. (g) The proposed mechanism for cocrystal formation. The pictures are reprinted with permission from Ref. [31]. Copyright 2015, John Wiley \& Sons, Inc. 
that of P3T1 cocrystals, since the mixed-like packing structure in P3T1 is not favourable for exciton separation. These interesting results here pave the way for rational design and preparation of cocrystals with desired functions. It should be noticed, because of the limited reports on vapor-phase co-crystallization and lack of mechanism investigation on mechanochemical method, further research should focus on these issues as well as the extension of organic cocrystals into two-dimensional (2D) structures [32].

Another long-standing problem is to understand the CT interactions and exciton dynamics. It is key important to confirm whether the CT interactions exist in cocrystal, which significantly affect the lattice instability [33] and bulk optoelectronic properties [34,35]. A puzzle we are encountering is to distinguish the ground and excited state [36,37]. Recently, we [38] found that both of ground and excited states of 1,2,4,5-tetracyanobenzene (TCNB) based cocrystals are actually CT state, as experimentally verified by spectroscopic studies, electron spin resonance (ESR) measurements and theoretical calculations (Fig. 2). Significantly, we demonstrated that the CT interactions in cocrystals are related to their molecular packing and can be a
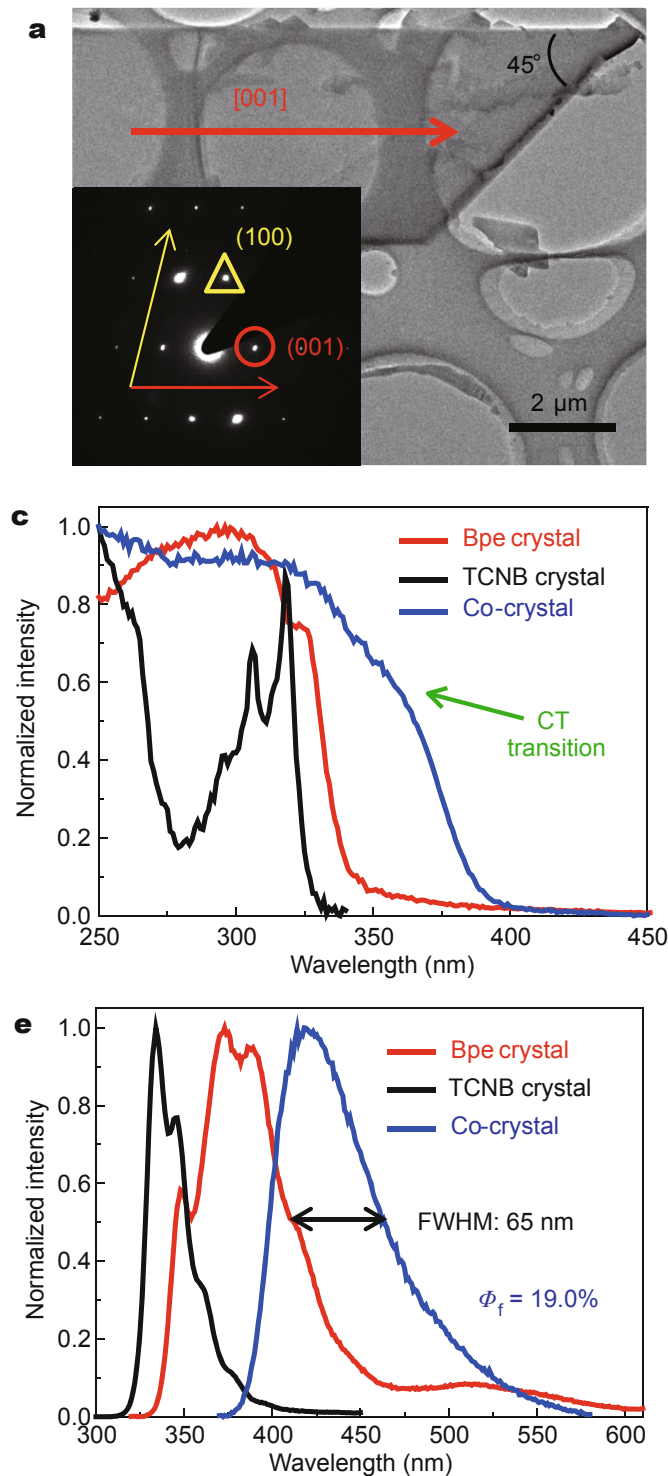

b
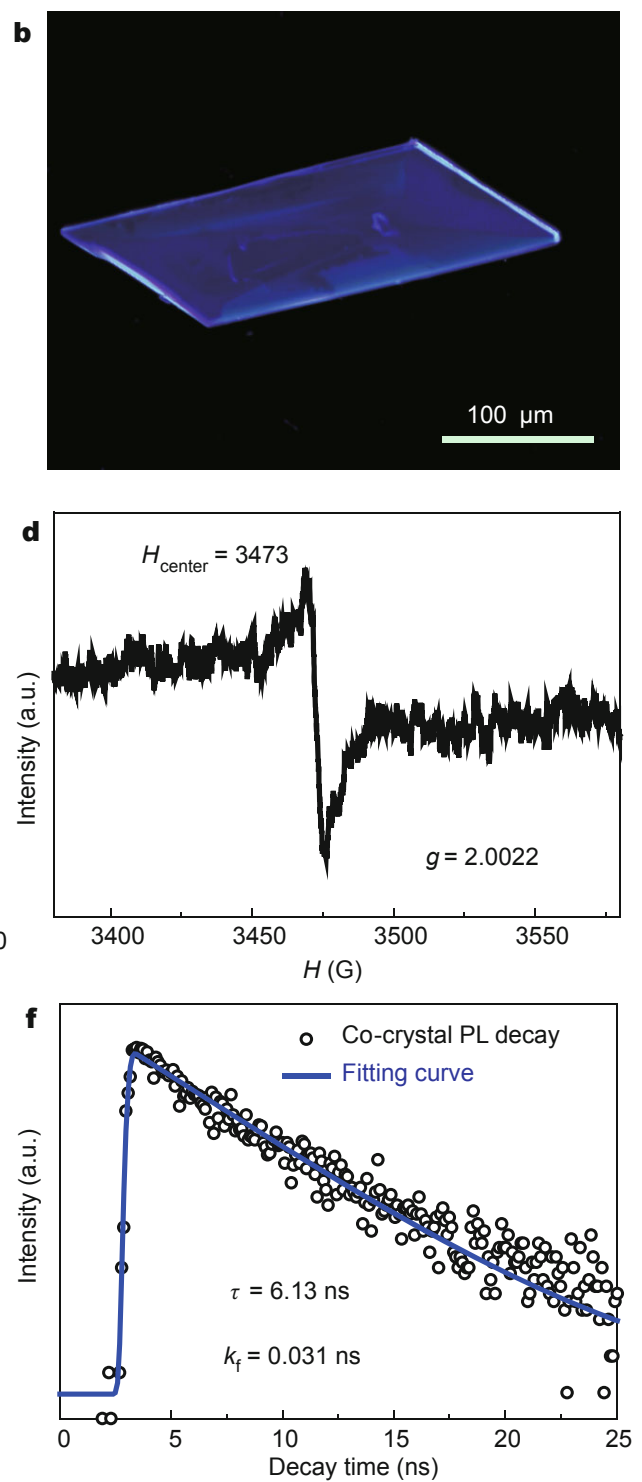

Figure 2 (a) The morphology and structure of Bpe-TCNB cocrystal. Bpe =1,2-di(4-pyridyl)ethylene. (b) The confocal laser scanning microscope image of cocrystal. (c) The absorption spectra, (d) electron spin resonance spectrum, (e) photoluminescence (PL) spectra, and (f) PL lifetime measurement of cocrystals. The pictures are reprinted with permission from Ref. [38]. Copyright 2015, John Wiley \& Sons, Inc. 
triggered or suppressed by crystal engineering [39], thus resulting in distinct optoelectronic properties. And we proposed that the $\pi$-electron-rich circumstances in molecular columns of donor are helpful to promote the CT process from donor to acceptor.

In order to gain a deeper understanding of fantastic optoelectronics, the dynamics of excitons in cocrystals should be concerned, especially in those with a neutral ground but a CT excited state [24]. In these cocrystals, the generated singlet excitons are changed ultrafast into CT excitons, which is common but largely puzzled in organic photovoltaics [40]. Due to the limited studies on these, further investigations may be devoted to revealing the exciton dynamics and related molecular dynamics by applying advanced time-resolved spectroscopic techniques, as well as theoretical calculations. Moreover, both of the CT degree in the ground state and its influences on physicochemical properties are not yet well understood.

The third challenge is to explore new physicochemical properties and potential applications for organic cocrystals. Since the discovery of high conductivity in 1973 [1,2], the research on this area has gained enormous attention, but now the question is what kind of new properties which have not been revealed in today's molecular materials that co-crystallization will bring to us, and what kind of novel application could be expected. Like organic-inorganic perovskites $[41,42]$, organic cocrystals are expected possessing not only ideal field-effect properties, but also photovoltaic, light-emitting and even magnetic properties. In our opinion, further research interests should mainly focus on ferroelectrics, nonlinear optics (such as two-photon absorption and fluorescence) and magnetic properties, as well as multifunctional and smart cocrystal materials. We believe that organic cocrystal is at the fundamental heart of molecular science and is now gaining a lot of attention. And we believe, in the near future, more and more research on this area will take us to a beautiful and fantastic "cocrystal world".

Received 13 September 2015; accepted 13 November 2015; published online 18 November 2015

1 Coleman LB, Cohen MJ, Sandman DJ, et al. Superconducting fluctuations and the peierls instability in an organic solid. Solid State Commun, 1973, 12: 1125-1132

2 Ferraris J, Cowan DO, Walatka V, et al. Electron transfer in a new highly conducting donor-acceptor complex. J Am Chem Soc, 1973, 95: 948-949

3 Zhang J, Geng H, Virk TS, et al. Sulfur-bridged annulene-TCNQ co-crystal: a self-assembled "molecular level heterojunction" with air stable ambipolar charge transport behavior. Adv Mater, 2012, 24: $2603-2607$

4 Kang SJ, Kim JB, Chiu CY, et al. A supramolecular complex in small-molecule solar cells based on contorted aromatic molecules. Angew Chem Int Ed, 2012, 51: 8594-8597

5 Kang SJ, Ahn S, Kim JB, et al. Using self-organization to control morphology in molecular photovoltaics. J Am Chem Soc, 2013, 135: 2207-2212

6 Tremblay NJ, Gorodetsky AA, Cox MP, et al. Photovoltaic universal joints: ball-and-socket interfaces in molecular photovoltaic cells. Chemphyschem, 2010, 11: 799-803

7 Lei YL, Jin Y, Zhou DY, et al. White-light emitting microtubes of mixed organic charge-transfer complexes. Adv Mater, 2012, 24: 5345-5351

8 Bolton O, Lee K, Kim HJ, et al. Activating efficient phosphorescence from purely organic materials by crystal design. Nat Chem, 2011, 3: $205-210$

9 Mazumdar S, Guo F, Meissner K, et al. A new class of collective excitations: exciton strings. J Chem Phys, 1996, 104: 9283-9291

10 Rao SM, Batra AK, Lal RB, et al. Mixed methyl-(2,4-dinitrophenyl)-aminopropanoate: 2-methyl-4-nitroaniline crystal-a new nonlinear optical material. J Appl Phys, 1991, 70: 6674-6678

11 Zyss J, Ledoux-Rak I, Weiss HC, et al. Coupling octupoles in crystals: the case of the 1,3,5-trinitrobenzene-triphenylene 1:1 molecular co-crystal. Chem Mater, 2003, 15: 3063-3073

12 Bosshard C, Wong MS, Pan F, et al. Self-assembly of an acentric co-crystal of a highly hyperpolarizable merocyanine dye with optimized alignment for nonlinear optics. Adv Mater, 1997, 9: 554-557

13 Horiuchi S, Ishii F, Kumai R, et al. Ferroelectricity near room temperature in co-crystals of nonpolar organic molecules. Nat Mater, 2005, 4: 163-166

14 Nguyen HL, Horton PN, Hursthouse MB, et al. Halogen bonding: a new interaction for liquid crystal formation. J Am Chem Soc, 2003, 126: $16-17$

15 Reczek JJ, Villazor KR, Lynch V, et al. Tunable columnar mesophases utilizing $C_{2}$ symmetric aromatic donor-acceptor complexes. J Am Chem Soc, 2006, 128: 7995-8002

16 Braga D, Maini L, Grepioni F. Mechanochemical preparation of co-crystals. Chem Soc Rev, 2013, 42: 7638-7648

17 Wöhler F. Untersuchungen über das Chinon. Annalen, 1844, 51: 153

18 Davey R, Garside J. From Molecules to Crystallizers. An Introduction to Crystallization. Oxford: Oxford University Press, 2000

19 Patrick CR, Prosser GS. A molecular complex of benzene and hexafluorobenzene. Nature, 1960, 187: 1021-1021

20 Desiraju GR. Crystal and co-crystal. CrystEngComm, 2003, 5: 466-467

21 Dunitz JD. Crystal and co-crystal: a second opinion. CrystEngComm, 2003, 5: 506

22 Lucassen ACB, Karton A, Leitus G, et al. Co-crystallization of sym-triiodo-trifluorobenzene with bipyridyl donors: consistent formation of two instead of anticipated three N...I halogen bonds. Cryst Growth Des, 2007, 7: 386-392

23 Harris KDM, Stainton NM, Callan AM, et al. Crystal engineering of hydrogen-bonded co-crystals between cyanuric acid and 'diamide' molecules. Investigations on the formation and structure of co-crystals containing cyanuric acid and oxalyl dihydrazide. J Mater Chem, 1993, 3: 947-952

24 Zhang J, Tan JH, Ma ZY, et al. Fullerene/sulfur-bridged annulene cocrystals: two-dimensional segregated heterojunctions with ambipolar transport properties and photoresponsivity. J Am Chem Soc, 2012, 135: 558-561

25 Boyd PDW, Reed CA. Fullerene-porphyrin constructs. Acc Chem Res, 2004, 38: 235-242

26 Kobayashi H, Nakayama J. The crystal structure of the charge-transfer complex of dibenzotetrathiafulvalene-tetracyanoquinodimeth- 
ane, DBTTF-TCNQ. B Chem Soc Jpn, 1981, 54: 2408-2411

27 Mahns B, Kataeva O, Islamov D, et al. Crystal growth, structure, and transport properties of the charge-transfer salt picene/2,3,5,6-tetrafluoro-7,7,8,8-tetracyanoquinodimethane. Cryst Growth Des, 2014, 14: 1338-1346

28 Black HT, Perepichka DF. Crystal engineering of dual channel p/n organic semiconductors by complementary hydrogen bonding. Angew Chem Int Ed, 2014, 53: 2138-2142

29 Li J, Takaishi S, Fujinuma N, et al. Enhancement of luminescence intensity in TMPY/perylene co-single crystals. J Mater Chem, 2011, 21: $17662-17666$

30 Friščić T, Jones W. Recent advances in understanding the mechanism of cocrystal formation via grinding. Cryst Growth Des, 2009, 9: 1621-1637

31 Zhu W, Yi Y, Zhen Y, et al. Precisely tailoring the stoichiometric stacking of perylene-TCNQ co-crystals towards different nano and microstructures with varied optoelectronic performances. Small, 2015, 11: 2150-2156

32 Huang X, Zhang H. Molecular crystals on two-dimensional van der Waals substrates. Sci China Mater, 2015, 58, 5-8

33 D'Avino G, Verstraete MJ. Are hydrogen-bonded charge transfer crystals room temperature ferroelectrics? Phys Rev Lett, 2014, 113: 237602

34 Dillon RJ, Bardeen CJ. The effects of photochemical and mechanical damage on the excited state dynamics of charge-transfer molecular crystals composed of tetracyanobenzene and aromatic donor molecules. J Phys Chem A, 2011, 115: 1627-1633

35 Torrance JB. The difference between metallic and insulating salts of tetracyanoquinodimethane (TCNQ): how to design an organic metal. Acc Chem Res, 1979, 12: 79-86

36 Bandrauk AD, Truong KD, Carlone C. Optical and Raman spec- tra of single crystals of perylene-TCNQ charge transfer complexes. Can J Chem, 1982, 60: 588-595

37 Bandrauk AD, Truong KD, Salares VR, et al. Raman spectra of solid perylene-TCNQ complexes. J Raman Spectrosc, 1979, 8: 5-10

38 Zhu W, Zheng R, Fu X, et al. Revealing the charge-transfer interactions in self-assembled organic cocrystals: two-dimensional photonic applications. Angew Chem Int Ed, 2015, 54: 6785-6789

39 Zhu W, Zheng R, Zhen Y, et al. Rational design of charge-transfer interactions in halogen-bonded co-crystals toward versatile solid-state optoelectronics. J Am Chem Soc, 2015, 137: 11038-11046

40 Hains AW, Liang ZQ, Woodhouse MA, et al. Molecular semiconductors in organic photovoltaic cells. Chem Rev, 2010, 110: 6689-6735

41 Qin X, Dong HL, Hu WP. Green light-emitting diode from bromine based organic-inorganic halide perovskite. Sci China Mater, 2015, 58: $186-191$

42 Gao Y, Wang JJ, Wu L, et al. Tunable magnetic and electrical behaviors in perovskite oxides by oxygen octahedral tilting. Sci China Mater, 2015, 58: 302-312

Acknowledgements This work was supported by the National Natural Science Foundation of China (91222203, 91233205, 51222306, 61201105, 91027043, 91433115, 51303185, 21473222, and TRR61), the Ministry of Science and Technology of China (2011CB808400, 2011CB932300, 2013CB933403, 2013CB933500, and 2014CB643600), and the Chinese Academy of Sciences.

Author contributions Zhu W, Dong H, Zhen Y and Hu W wrote the paper and contributed to the general discussion.

Conflict of interest The authors declare that they have no conflict of interest. 


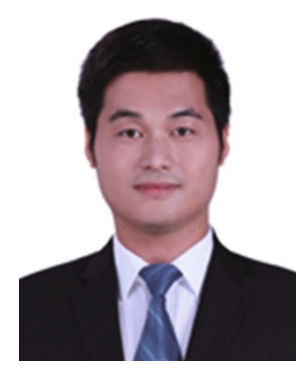

Weigang Zhu was born in 1988. He is now a PhD candidate in the Laboratory of Organic Solids, Institute of Chemistry, Chinese Academy of Sciences (ICCAS). His research interest is design, synthesis and applications of organic cocrystals, including: (1) basic nucleation and growth mechanism of cocrystals and related controlled self-assembling; (2) advanced physicochemical properties of cocrystals, such as ambipolar charge transport, white-light emitting, lasering, non-linear optics, ferroelectrics and spintronics; (3) ultrafast exciton and molecular dynamics in cocrystals.

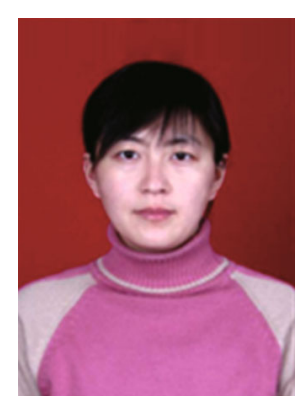

Huanli Dong is an associate professor of the ICCAS. She grew up in Shandong province, China. She received her $\mathrm{PhD}$ degree from the ICCAS in 2009 after she got her MSc degree in Fujian Institute of Research on the Structure of Material, Chinese Academy of Sciences in 2006. She is presently focusing on the self-assembling of molecular materials and the application of molecular materials in optoelectronic devices, and she has more than 90 refereed publications with citation over 2500 times $(\mathrm{H}$ index=22).

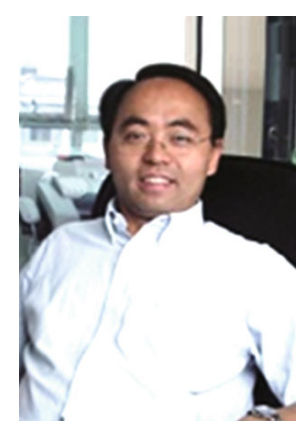

Wenping Hu is a professor of the ICCAS. He received his PhD from the ICCAS in 1999. He then joined Osaka University as a research fellow of Japan Society for the Promotion of Sciences and Stuttgart University as an Alexander von Humboldt. In 2003 he worked for Nippon Telephone and Telegraph, and then returned to ICCAS and was promoted as full professor. His research focuses on molecular electronics and he has more than 350 refereed publications with citation over 10000 times $(\mathrm{H}$ index=53).

中文摘要 本文简明扼要地介绍了“有机共晶”这个重要研究领域的兴起和发展过程, 在有争议的方面, 如共晶定义和如何判定分子排 布等, 给出了作者独到的见解. 特别重要的是, 通过阐述该领域当前存在的问题和挑战, 结合分析最新的研究进展和结果, 使得人们对共 晶的认识更为系统和深入. 基于此, 作者同时分析和指出了未来可能重点发展的研究方向. 